\title{
Bacteraemia after leadless pacemaker implantation
}

Christophe Garweg, MD ${ }^{1,2}$, Bert Vandenberk, MD, PhD ${ }^{1}$, Sander Jentjens, M.D. ${ }^{3}$, Stefaan Foulon ${ }^{1}$, Patrick Hermans ${ }^{1}$, Patricia Poels ${ }^{1}$, Peter Haemers $\mathrm{MD}, \mathrm{PhD}^{1,2}$ Joris Ector $\mathrm{MD}, \mathrm{PhD}^{1,2}$ and Rik Willems, MD, $\mathrm{PhD}^{1,2}$

1. University Hospitals Leuven, Cardiology

2. University of Leuven, Department of Cardiovascular Sciences

3. University Hospitals Leuven, Nuclear Medicine

Short title: Bacteraemia and leadless pacemakers

\section{Corresponding author:}

Christophe Garweg, M.D., Cardiology, Herestraat 49, 3000 Leuven, Belgium

Tel: +3216342079

Email: christophe.garweg@uzleuven.be

Word count: 2192 (including the abstract). Abstract only: 250 words

Contributors: All the authors contributed significantly to the completion of the study and the manuscript, including reading and approval of the manuscript in its current form.

Funding: None

Author disclosure statement: RW/JE/PH/CG research funding: Biotronik, Boston Scientific, Medtronic. Speakers and consultancy fees: Medtronic, Boston Scientific, Biotronik, Abbott, Microport. CG was supported as predoctoral clinical researcher by the Fund for Scientific Research Flanders. RW/JE are supported as postdoctoral clinical researcher by the Fund for Scientific Research Flanders. 


\section{Abstract}

Background: Transvenous permanent pacemaker related infection is a severe condition associated with significant morbidity and mortality. Leadless pacemakers may be more resistant to bacterial seeding during bloodstream infection because of its small surface area and encapsulation in the right ventricle. This study reports the incidence and outcomes of bacteraemia in patients implanted with a Micra leadless pacemaker. We present ${ }^{18} \mathrm{~F}$-FDG PET/CT findings obtained in a sub-group of patients.

Methods: We report a retrospective cohort study of 155 patients who underwent a Micra TPS implant procedure at the University Hospitals of Leuven between July 2015 and July 2019. We identified the patients who developed an episode of bacteraemia, proved by $\geq 2$ positive blood cultures.

Results: Of the 155 patients, 15 patients presented an episode of bacteraemia at a median of 226 days (range: 3-1129) days after the implant procedure. Gram positive species accounted for $73.3 \%(n=11)$ of the bacteraemia including Staphylococcus $(n=5)$, Enterococcus $(n=3)$ and Streptococcus $(n=3)$. The source of infection was identified in 9 patients $(60 \%)$ including endocarditis in 4 patients, urinary tract in 3 patients, and skin in 2 patients. ${ }^{18} \mathrm{~F}$-FDG PET/CT imaging performed in 6 patients did not show sign of infection around the leadless pacemaker. Bacteraemia was resolved in all patients after adequate antibiotherapy. Four patients died early during follow up. For all other patients, there were no recurrence of systemic infection during a median follow up of 263 days (range 15-1134).

Conclusion: In our small cohort, no leadless pacemaker endocarditis was observed among patients with bacteraemia.

Keyword: leadless pacemaker, infection, bacteraemia, ${ }^{18} \mathrm{~F}$-FDG PET/CT 


\section{Introduction}

The infection of cardiac implantable electronic devices (CIEDs) is a feared and devastating complication associated with a high morbidity, mortality and healthcare costs ${ }^{1,2}$. Removal of the infected device and extraction of the leads followed by prolonged administration of intravenous antibiotics are the core of the treatment. The incidence of CIEDs infection ranges from 0.5 to $2.2 \%$ for permanent pacemakers and implantable cardiac defibrillators ${ }^{3,4}$. In the setting of bacteraemia, the risk for CEID infection depends on the offending species. Staphylococcus aureus carries a higher risk of device involvement as compared to gram negative bacilli except with Pseudomonas aeruginosa and Serratia marscecens $s^{5,6}$. While diagnosis of CIED pocket infection can be generally confirmed by local inflammatory signs, the diagnosis of lead infection may be more challenging. To improve the diagnostic yield of CIED infection, the European Society of Cardiology and Heart Rhythm Society have suggested that the use of ${ }^{18} \mathrm{~F}$ fluorodeoxyglucose (FDG)- PET/CT imaging might provide helpful evidence for inconclusive cases in their guidelines ${ }^{1,7}$. The uptake of ${ }^{18} \mathrm{~F}-\mathrm{FDG}$, a glucose analogue, by the leukocytes has a high sensitivity in detecting infection using PET imaging and the combination of PET/CT imaging in recent systems provide the location of infectious foci with high spatial resolution ${ }^{8-10}$.

Leadless pacing system has been recently introduced for patients requiring ventricular pacing to overcome pocket and lead-related complications ${ }^{11}$. The updated results of the Micra Post-Approval Registry consistently showed the safety and the efficacy of leadless pacing with lower rates of complications including device infection as compared to a historical patients cohort with conventional permanent pacing ${ }^{12}$. Leadless pacemakers may be more resistant to bacterial seeding during bloodstream infection because of their intrinsic characteristics including a small surface area and encapsulation in the right ventricle $\mathrm{e}^{11,13,14}$. In the present study, we report the incidence and outcome of bacteraemia in patients implanted with a Micra Transcatheter Pacing System (TPS) (Medtronic, Minneapolis, MN, US) in our institution. We also present ${ }^{18} \mathrm{~F}$-FDG PET/CT findings obtained in a patients sub-group. 



\section{Methods}

\section{Patient Cohort}

We conducted a retrospective study which examined all patients aged over 18 years old with class I or II guideline recommendations for ventricular pacing who underwent a successful Micra TPS implantation in our centre between July 2015 and July 2019 ${ }^{15}$. Patients data were collected from our electronic institutional database. We searched bacteriological reports to identify all patients with positive blood culture. Bacteraemia was confirmed in the presence of at least 2 positive peripheral blood cultures with elevated white blood cell count. We identified patients who underwent ${ }^{18} \mathrm{~F}$-FDG $\mathrm{PET} / \mathrm{CT}$ imaging in the setting of the bacteraemia. The protocol was approved by the Ethics Committee of the University Hospitals of Leuven.

\section{${ }^{18}$ F-FDG PET/CT protocol}

The acquisition of the PET/CT scan was performed within 2 weeks after diagnosis of the bacteraemia. PET-CT scans were performed on a Biograph Truepoint 40 (Siemens, Erlangen, Germany) or Discovery 670 MI (General Electric Healthcare, Milwaukee, WI, USA). PET imaging was performed 45-60 minutes after the administration of $4.5 \mathrm{MBq} / \mathrm{kg}$ of ${ }^{18} \mathrm{~F}-\mathrm{FDG}$. Patients were instructed to use a carbohydrate poor, fat and protein rich diet for $24 \mathrm{~h}$ prior to suppress the physiological glucose metabolism of the myocytes and in addition fast for a minimum of 6 hours prior to injection. Blood glucose levels were controlled and below $180 \mathrm{mg} / \mathrm{dL}$ at the time of intravenous ${ }^{18} \mathrm{~F}-\mathrm{FDG}$ administration. Patients received $20 \mathrm{mg}$ Propranolol hydrochloride to minimise uptake in brown fat.

\section{${ }^{18}$ F-FDG PET/CT images analysis}

CT staging was performed using contrast-enhanced high dose CT images obtained from the PET/CT scanner. CT images were interpreted independently by an experienced radiologist. The PET part of the $\mathrm{PET} / \mathrm{CT}$ was analysed by experienced nuclear medicines. Attenuation-corrected as well as nonattenuation-corrected images were reviewed to avoid misinterpretation of attenuation-correction 
artefacts due to the proximity of dense materials (e.g. device related). Qualitative analysis of the images was performed by two nuclear physicians identifying the areas with increased ${ }^{18} \mathrm{~F}$-FDG uptake. The presence of increased ${ }^{18}$ F-FDG activity that could not be explained by physiological uptake was considered as a potential site of inflammation. PET and CT findings were integrated in a final hybrid PET/CT finding. As previously described by Sarrazin et al., a semi-quantitative evaluation of ${ }^{18} \mathrm{~F}$-FDG uptake by the leadless device was also performed : a ratio between the maximum count rate of the device over a mean count rate between normal right and left lung parenchyma referred a semiquantitative ratio (SQR) above 1.87 was considered positive for device infection ${ }^{8}$.

\section{Statistics}

Data collected in all patients after Micra TPS implantation were included in the statistical analysis. Continuous variables were tested for normal distribution by the Kolmogorov-Smirnov test. Since all parameters deviated significantly from the normal distribution, data were summarized using median and interquartile ranges, unless specified otherwise. Categorical variables were presented as numbers and percentages. Continuous variables were compared between the groups using the non-parametric Mann-Whitney-U testing. Categorical variables were compared using the chi-square test. P-values $<0.05$ were considered significant. All statistical analyses were performed using SPSS (IBM statistics, version 25).

\section{Results}

A total of 155 patients underwent a Micra TPS implant procedure from July 2015 to July 2019 of which 15 patients (9.7\%) developed a bacteraemia during follow-up. The baseline characteristics of the population is summarized in Table 1. Patients with bacteraemia had a median age of 81-years (77-82) and were largely similar to the other patients with two exceptions. First, they had a more frequent history of valve intervention (66.7\% vs.27.9\%, $\mathrm{p}=0.002)$ and secondly, there was a higher incidence of severe renal dysfunction requiring haemodialysis ( $20 \%$ vs. $3.6 \%, p=0.006)$. 
Bacteraemia occurred at a median of 226 days (range: 3-1129) after the implant procedure (patients details in Table 2). Median duration of follow-up after the bacteraemia was 263 days (range: 15-1134). Gram positive species accounted for $73.3 \%(n=11)$ of the bacteraemia including Staphylococcus $(n=5)$, Enterococcus $(n=3)$ and Streptococcus $(n=3)$. The source of infection was identified in 9 patients $(60 \%)$ including endocarditis in 4 patients, the urinary tract in 3 patients, and the skin in 2 patients. All patients underwent at least one transoesophageal echocardiography that did not reveal the presence of vegetation on the Micra. Thirteen patients were successfully treated with intravenous antibiotics. Two patients with endocarditis underwent valvular replacement with preventive removal of the Micra in the absence of definite signs of device infection including negative transoesophageal echocardiography. In both, the culture of the explanted device remained negative. All treatments led to negative conversion of the blood cultures and no patient experienced any recurrent systemic infection or bacteraemia during follow-up (Table 2). Four patients died during follow-up of which 3 patients with endocarditis. Both patients who had removal of the Micra with valve replacement for endocarditis died of multi-organ failure respectively 14 and 43 days after surgery. The third patient also died of multi-organ failure 20 days after valve surgery for endocarditis. A fourth patient died at home from causes unrelated to the bacteraemia.

Two patients (patients 13 and 14) had an episode of bacteraemia shortly after the Micra implantation procedure (<30 days). In patient 13 who was implanted with a leadless pacemaker due to a complete atrioventricular block after valve replacement for mitral valve endocarditis, the bacteraemia was not related to the Micra implant procedure but to the early recurrence of endocarditis on the mitral valve prosthesis. In patient 14 with previous extraction of an infected pacemaker, the bacteraemia was related to the development of a skin infection at the femoral puncture site for the Micra implant procedure.

${ }^{18}$ FDG PET/CT imaging was performed in 6 patients to identify the site of infection. Based on the independent qualitative assessment by 2 nuclear physicians, no patient had abnormal ${ }^{18} \mathrm{~F}$-FDG uptake 
detected around the Micra on the PET/CT fusion images (Figure 1). Two patients with endocarditis had increased ${ }^{18} \mathrm{~F}$-FDG uptake on infected valves confirmed by positive post-operative valve culture. Two other patients had increased focal ${ }^{18}$ F-FDG uptake compatible with infectious arthritis (Table 2). Using semi-quantitative analysis according to Sarrazin, 2 patients had a SQR $>1.87$ without recurrence of sign of infection in the absence of device extraction.

After the episode of bacteraemia, all surviving patients $(n=12)$ had clinical follow up with regular laboratory test (including white blood cell count, neutrophils count, and C-reactive protein level). Blood cultures performed during and up to one month after antibiotherapy were negative. Repeated blood cultures in 3 patients for later recurrence of fever with unknown origin also remained negative. . Details of the patients' biological follow-up are reported in Table 2.

\section{Discussion}

Our study supports the hypothesis that leadless devices are less prone to haematogenous pacemaker infections. In our population, the rate of bacteraemia was almost $10 \%$ suggesting that this was a highrisk group. One of the most relevant finding of the study is that patients who were previously implanted with a leadless pacemaker, do not seem to require systematic pacemaker removal in case of bacteraemia.

Haematogenous seeding of CIED during bacteraemia is a severe condition that is associated to significant morbidity and mortality if unsuccessfully managed ${ }^{1,16}$. In case of pacemaker infection, the complete removal of the hardware is a clear indication in the guidelines ${ }^{2,7}$. Staphylococcus aureus bacteraemia has been associated with a high risk of cardiac device related infection ${ }^{5}$. This is a result of the high propensity of the pathogen to adhere to foreign materiel implanted in the body including pacemaker leads ${ }^{17}$.

As leadless pacing technology is relatively new in clinical use, there are only few reports on the risk of device infection ${ }^{18,19}$. In our study, none of the patients had definite sign of Micra infection in the setting of or after bacteraemia even involving gram positive Staphylococcus. In 2 cases, the absence of device 
infection was confirmed by negative culture of the device following its preventive removal during valve surgery for endocarditis while there was no sign of device infection on preoperative transoesophageal echocardiographic images for both patients and on additional ${ }^{18}$ FDG PET/CT imaging for one of the patients. Overall, the absence of device infection in our series is also ascertained by the absence of recurrent systemic infection or bacteraemia reported during the follow up after antibiotic treatment and surgery when appropriate. Our results are in accordance with those reported by El Chami et al. for patients with systemic infection from the Micra IDE study ${ }^{18}$. Among 720 patients implanted with a Micra, the authors reported the occurrence of 21 serious infectious events (bacteraemia or endocarditis) in 16 patients that were successfully treated with antibiotics without evidence of leadless pacemaker infection during follow up.

For the first time, this study reported on the use of ${ }^{18} \mathrm{~F}$-FDG/PET imaging to rule out leadless pacemaker infection during bacteraemia. In this small series of 6 patients, the qualitative analysis of ${ }^{18} \mathrm{~F}-\mathrm{FDG} / \mathrm{PET}$ images did not identify leadless device infection. Discordant results were observed for 2 patients using the semi-quantitative analysis proposed by Sarrazin for the detection of an infection in the presence of conventional pacemaker. However, those 2 patients who were treated based on qualitative evaluation of ${ }^{18} \mathrm{FDG}$ PET images had an uneventful course during follow up. Therefore, it is uncertain whether Sarrazin's semi-quantitative analysis can be applied for the detection of leadless pacemaker infection ${ }^{8}$. So far, the European Association of Nuclear Medicine recommends a qualitative evaluation of ${ }^{18} \mathrm{~F}$-FDG PET images in the case of infectious endocarditis and CIED infections ${ }^{20}$. Semi-quantitative analysis using standard uptake value (SUV) that is applied in oncology has not yet been validated either in the diagnosis of CEID infection.

The absence of Micra related infection detected during bacteraemia, even when the bacteraemia occurs early after the implant procedure, is reassuring. First, it might be explained by the intrinsic characteristics of the device: the use of a parylene coating seems to have protective effect against bacterial grow and adhesion while its reduced size with a small surface area in contact with the 
bloodstream might also be protective against bacterial seeding ${ }^{11,21}$. Secondly, the reported progressive fibrous encapsulation of the device could also contribute to reduce the risk of infection. Autopsies have revealed partial device encapsulation as early as 2 months after implantation and complete encapsulation at 1 year ${ }^{13,14}$. Finally, the absence of a pacemaker pocket and the use of a catheter delivery system avoids all manual manipulation of the device during the implant procedure and are also potential factors contributing to the reduction of the risk of bacterial seeding as compared to conventional pacemaker. In our series, this is illustrated by the occurrence of a skin infection at the femoral puncture site used for the Micra implant procedure without any involvement of the intracardiac device.

In our series, the mortality rate was high $(26.6 \%)$ with $75 \%$ mortality in patients with endocarditis.Previously, it has been shown that the 6-month mortality after an infective endocarditis was associated with eldery ( $>80$ years old), infection of prosthetic valve and the causative microorganism (Staphylococci and Enterococci) ${ }^{22}$. In our population, the 3 patients with endocarditis cumulated those risk factors.

Previous studies have identified host-related risk factors for CIED that include diabetes mellitus, end stage renal disease, chronic obstructive pulmonary disease, corticosteroid use, history of previous infection, renal insufficiency, malignancy, heart failure, pre-procedural fever, anticoagulant use and skin disorders ${ }^{1,3,23}$. In our case series, patients who developed bacteraemia after Micra implantation had a higher incidence of end-stage renal disease treated by dialysis and of previous valve intervention. The use of leadless pacemaker might be of interest in these patients, reducing the risk of device-related infection/endocarditis.

\section{Study limitations}

The single centre experience and the small number of patients included in the study are the main limitations of this study. The duration of the follow-up may also have influenced our results. Furthermore, we cannot exclude unreported serious infectious events that could have occurred in our 
patients during hospitalization in another institution. Another limitation to be considered is the absence of direct comparison with a group of patients receiving a conventional single chamber pacemaker.

\section{Conclusion}

In our cohort of patients, no leadless pacemaker endocarditis was observed after an episode of bacteraemia. This should be confirmed in large scale trials before setting recommendations about the preferential use of leadless pacemakers in patients with a high risk of infection. 


\section{References:}

1. Habib G, Lancellotti P, Antunes MJ, et al. 2015 ESC Guidelines for the management of infective endocarditis: The Task Force for the Management of Infective Endocarditis of the European Society of Cardiology (ESC). Endorsed by: European Association for Cardio-Thoracic Surgery (EACTS), the European Association of Nuclear Medicine (EANM). Eur Heart $J$. 2015;36(44):3075-3128.

2. Bongiorni MG, Burri H, Deharo JC, et al. 2018 EHRA expert consensus statement on lead extraction: recommendations on definitions, endpoints, research trial design, and data collection requirements for clinical scientific studies and registries: endorsed by APHRS/HRS/LAHRS. Europace. 2018;20(7):1217.

3. Olsen T, Jorgensen OD, Nielsen JC, Thogersen AM, Philbert BT, Johansen JB. Incidence of device-related infection in 97750 patients: clinical data from the complete Danish devicecohort (1982-2018). Eur Heart J. 2019;40(23):1862-1869.

4. Poole JE, Gleva MJ, Mela T, et al. Complication rates associated with pacemaker or implantable cardioverter-defibrillator generator replacements and upgrade procedures: results from the REPLACE registry. Circulation. 2010;122(16):1553-1561.

5. Chamis AL, Peterson GE, Cabell $\mathrm{CH}$, et al. Staphylococcus aureus bacteremia in patients with permanent pacemakers or implantable cardioverter-defibrillators. Circulation. 2001;104(9):1029-1033.

6. Maskarinec SA, Thaden JT, Cyr DD, Ruffin F, Souli M, Fowler VG. The Risk of Cardiac DeviceRelated Infection in Bacteremic Patients Is Species Specific: Results of a 12-Year Prospective Cohort. Open Forum Infect Dis. 2017;4(3):ofx132.

7. Kusumoto FM, Schoenfeld MH, Wilkoff BL, et al. 2017 HRS expert consensus statement on cardiovascular implantable electronic device lead management and extraction. Heart Rhythm. 2017;14(12):e503-e551.

8. Sarrazin JF, Philippon F, Tessier M, et al. Usefulness of fluorine-18 positron emission tomography/computed tomography for identification of cardiovascular implantable electronic device infections. J Am Coll Cardiol. 2012;59(18):1616-1625.

9. Graziosi M, Nanni C, Lorenzini M, et al. Role of (1)(8)F-FDG PET/CT in the diagnosis of infective endocarditis in patients with an implanted cardiac device: a prospective study. Eur J Nucl Med Mol Imaging. 2014;41(8):1617-1623.

10. Bensimhon L, Lavergne $T$, Hugonnet $F$, et al. Whole body [(18) F]fluorodeoxyglucose positron emission tomography imaging for the diagnosis of pacemaker or implantable cardioverter defibrillator infection: a preliminary prospective study. Clin Microbiol Infect. 2011;17(6):836844.

11. Reynolds D, Duray GZ, Omar R, et al. A Leadless Intracardiac Transcatheter Pacing System. $N$ Engl J Med. 2016;374(6):533-541.

12. El-Chami MF, Al-Samadi F, Clementy N, et al. Updated performance of the Micra transcatheter pacemaker in the real-world setting: A comparison to the investigational study and a transvenous historical control. Heart Rhythm. 2018;15(12):1800-1807.

13. Satoh T, Fukui A, Katoh S, Matsui M. A Leadless Pacemaker Which Became Encapsulated Only Two Months after Placement. Intern Med. 2018;57(20):3053-3054.

14. Kypta A, Blessberger $\mathrm{H}$, Lichtenauer $\mathrm{M}$, Steinwender $\mathrm{C}$. Complete encapsulation of a leadless cardiac pacemaker. Clin Res Cardiol. 2016;105(1):94.

15. Brignole M, Auricchio A, Baron-Esquivias G, et al. 2013 ESC Guidelines on cardiac pacing and cardiac resynchronization therapy: the Task Force on cardiac pacing and resynchronization therapy of the European Society of Cardiology (ESC). Developed in collaboration with the European Heart Rhythm Association (EHRA). Eur Heart J. 2013;34(29):2281-2329. 
16. Greenspon AJ, Rhim ES, Mark G, Desimone J, Ho RT. Lead-associated endocarditis: the important role of methicillin-resistant Staphylococcus aureus. Pacing Clin Electrophysiol. 2008;31(5):548-553.

17. Nagpal A, Baddour LM, Sohail MR. Microbiology and pathogenesis of cardiovascular implantable electronic device infections. Circ Arrhythm Electrophysiol. 2012;5(2):433-441.

18. El-Chami MF, Soejima K, Piccini JP, et al. Incidence and outcomes of systemic infections in patients with leadless pacemakers: Data from the Micra IDE study. Pacing Clin Electrophysiol. 2019;42(8):1105-1110.

19. El-Chami MF, Johansen JB, Zaidi A, et al. Leadless pacemaker implant in patients with preexisting infections: Results from the Micra postapproval registry. J Cardiovasc Electrophysiol. 2019;30(4):569-574.

20. Erba PA, Lancellotti P, Vilacosta I, et al. Recommendations on nuclear and multimodality imaging in IE and CIED infections. Eur J Nucl Med Mol Imaging. 2018;45(10):1795-1815.

21. El-Chami MF, Mayotte J, Bonner M, Holbrook R, Stromberg K, Sohail MR. Reduced bacterial adhesion with parylene coating: Potential implications for Micra transcatheter pacemakers. $J$ Cardiovasc Electrophysiol. 2020.

22. Hill EE, Herijgers $P$, Claus $P$, Vanderschueren $S$, Herregods $M C$, Peetermans WE. Infective endocarditis: changing epidemiology and predictors of 6-month mortality: a prospective cohort study. Eur Heart J. 2007;28(2):196-203.

23. Polyzos KA, Konstantelias AA, Falagas ME. Risk factors for cardiac implantable electronic device infection: a systematic review and meta-analysis. Europace. 2015;17(5):767-777. 


\section{Figure legend:}

Figure 1: Example of ${ }^{18}$ F-FDG PET/CT images in a 74-year-old-man (patient 15) with an infective endocarditis of a bioprosthetic aortic valve and native mitral valve with positive blood culture for Staphylococcus lugdunensis. Micra TPS was implanted 3 months before the infectious event. Columns $A$ and $B$ show images centred on aortic prosthesis (white arrow) and mitral valve (yellow arrow). $\mathrm{PET} / \mathrm{CT}$ images show an increased ${ }^{18} \mathrm{~F}-\mathrm{FDG}$ uptake in the region of the prosthetic aortic valve and native mitral valve. Columns $C$ and $D$ show images centred on the Micra leadless pacemaker. PET/CT fusion images show no increased ${ }^{18} \mathrm{~F}$-FDG uptake around the device 